\title{
Sequencing and assembly of transcriptome in non-photosynthetic plant Lathraea squamaria and its dynamics during the light and dark period of the day
}

\author{
E.S. Gerasimov ${ }^{1,3}$, A.A. Penin ${ }^{1,3}$ \\ ${ }^{1}$ Lomonosov Moscow State University, Moscow, Russia \\ ${ }^{2}$ Skolkovo Institute of Science and Technology, Moscow, Russia \\ ${ }^{3}$ Institute for Information Transmission Problems, Moscow, Russia \\ ${ }^{4}$ Vavilov Institute of General Genetics, Moscow, Russia \\ *maria.log@gmail.com
}

M.D. Logacheva ${ }^{1,2 *}$, A.V. Klepikova ${ }^{3}$, M.I. Schelkunov ${ }^{3}$, A.S. Kasianov ${ }^{4}$,

Key words: non-photosynthetic plants, RNA-seq, circadian clock

Motivation and Aim: Photosynthesis is one of the most prominent features of plants. However, several species have adapted to parasitic lifestyle and lost ability to photosynthesis. Currently little is known about the genomic changes that mediate this adaptation. We studied this using as a model Lathraea squamaria, parasitic plant from the family Orobanchaceae, with focus on genetic networks that regulate plant interaction with light. As we have previously shown, L. squamaria is characterized by rather recent switch to heterotrophy and thus allows inferring first stages of heterotrophy-related genome modification.

Methods and Algorithm: Plant material was collected in two replicates at 15 and 18 hours and then to the midnight with a 1-hour intervals. In order to represent non-polyadenylated transcripts total RNA after depletion of rRNA was used for library construction. Libraries were sequenced with $150+150$ bp length on Nextseq (Illumina) platform. Assembly and gene expression analysis was performed using CLC Genomics Workbench; transcripts were annotated using blast2go.

Results: As expected, the transcripts for most proteins involved in photosynthesis (components of photosystems I and II, cytochrome b6/f complex, light-harvesting complex, rubisco) are either absent or represented by expressed pseudogenes (in particular, plastid-encoded components of electron transfer chain). In contrast, the transcripts encoding proteins that are involved in non-photosynthetic interaction with light (e.g. phytochromes, cryptochromes, circadian clock associated proteins) are present. We found that several groups of genes significantly increase their expression after the sunset. Unexpectedly among them, there are the genes involved in cell division and translation.

Conclusion: The results obtained suggest that non-photosynthetic plants, even those that recently transitioned to heterotrophy, the genome undergoes profound changes that result in the loss (physical loss of gene or the downregulation of expression) of photosynthesisrelated genes. Other aspects of interaction with light, in particular those that are involved in photomorphogenesis, are likely to remain unchanged. Also, novel mechanisms, not typical for photosynthetic plants, exist in heterotrophic plants; they presumably reflect the interaction of parasite with its host plant. 